\title{
Finding Origin Points for New Coordinate System Suitable for Sign Animation
}

\author{
Tomohiro Kuroda ${ }^{1,2}$, Kazuya Okamoto ${ }^{2}$, Ryo Kitauchi ${ }^{3}$, \\ Tadamasa Takemura $^{1,2}$, Naoki Ohboshi ${ }^{3}$, and Hiroyuki Yoshihara ${ }^{1,2}$ \\ ${ }^{1}$ Department of Medical Informatics, Kyoto University Hospital, \\ 54 Kawahara-cho, Shogo-in, Sakyo-ku, Kyoto, 606-8507, Japan \\ ${ }^{2}$ Graduate School of Informatics, Kyoto University, \\ 36-1, Yoshida-Honmachi, Sakyo-ku, Kyoto, 606-8501, Japan \\ ${ }^{3}$ School of Science and Engineering, Kinki University, \\ 3-4-1, Kowakae, Higashi-Osaka, 577-8502, Japan \\ \{Tomohiro.Kuroda, kazuya, takemura\} @kuhp.kyoto-u.ac.jp, \\ stern@info.kindai.ac.jp, lob@kuhp.kyoto-u.ac.jp
}

\begin{abstract}
This paper proposes new coordinate system suitable for denoting sign language motion. As the proposed coordinate system consists of polar coordinate systems whose origins are certain points of human body, postures shown on the system can be proportional for avatars with any possible shape and fit with existing subjective sign notation systems. This paper extracted coordinate origins from Japanese-Japanese Sign Language Dictionary via morphological analysis. Selected 85 points are successfully mapped on $\mathrm{H}$ ANIM standard humanoid avatar.
\end{abstract}

Keywords: Japanese Sign Language, Natural Language Processing, Sign Language Dictionary, Sign Animation.

\section{Introduction}

Sign linguistic engineering is a group of research to develop communication aid (called sign information system) for the Deaf and the Hearing Impaired, who have communication barrier in social lives, using information technology [1]. Fig. 1 shows the general view of sign information systems. Any sign information systems, such as virtual reality based sign language telephones, automatic translation system between sign languages and phonetic languages, and even sign language dictionary, share same basic structure. Most of sign information systems use three-dimensional computer graphic model of human beings called avatar to show signs. To obtain signs, though several systems using image recognition technologies, most of them are utilizing motion capture systems consists of data-gloves and position and orientation sensors either magnetic-field-based, ultrasound-based, and image-based. The authors also developing sign language telephone named S-TEL [2] and consequent systems based on motion capture system consists of two data-gloves and three position and orientation sensors. 
Most of sign animations handles motion as sets of rotation angles of each joint and generate animation applying the data to an avatar model, just as same as MPEG-4 HANIM standard [3]. However, a certain body motion parameter to show a certain sign on a certain avatar cannot produce the same sign when it applied on another avatar with different body shape. Thus, the sign linguistic system needs to prepare an avatar, which holds the same body shape as a certain person to produce proper signs. Although authors developed a system to morph a standard avatar to fit a certain person through image recognition [4], it cannot give users full selection of avatar's outlook to have favorable view. Thus, to give flexibility and usability on sign information systems, a new motion coding system, which is independent from body shape and easily transferable from or to standard motion data such as a captured motion, data via a certain motion capture system or H-ANIM.

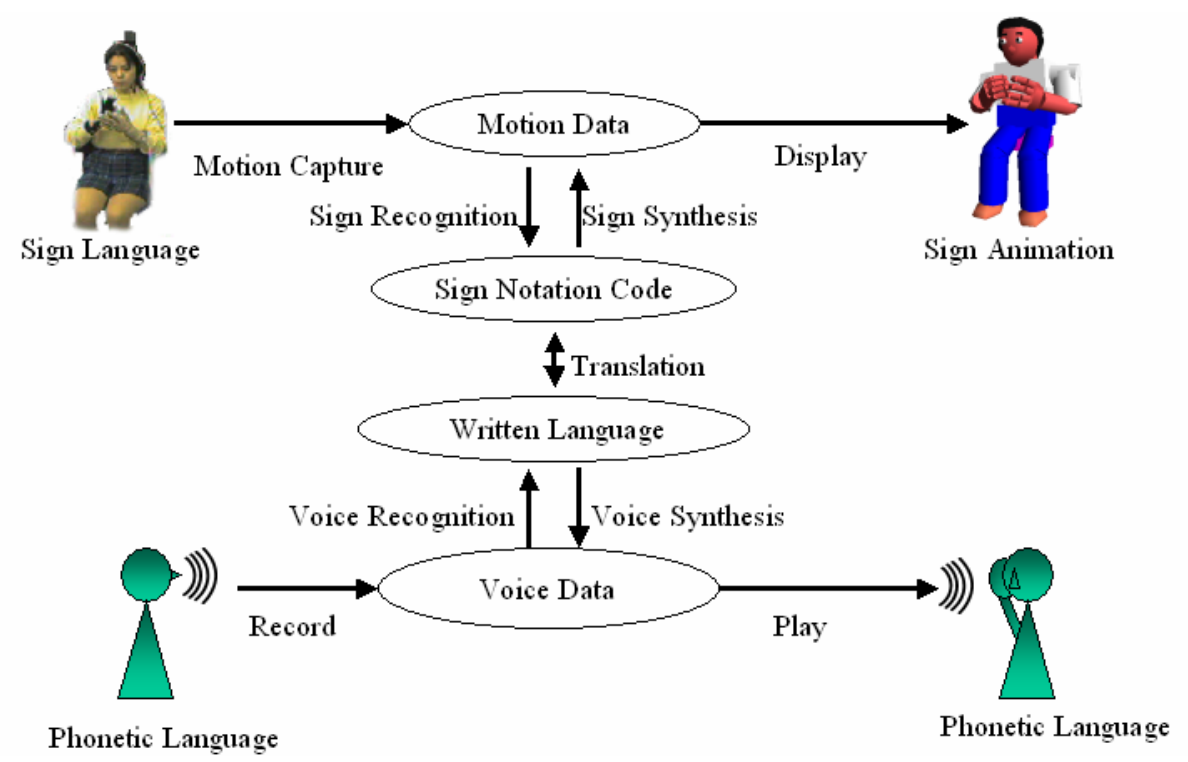

Fig. 1. General Overview of sign information systems

This paper proposes new coordinate system suitable for sign animation. Notations of textbooks or dictionaries of sign language present a position and orientation of hands in a certain posture in relation to a certain point of human body.

\section{Foregoing Coding Systems}

Foregoing researches on sign language developed several types of sign coding systems. Most of the sign notation system follows framework of Stokoe [5], which claims signs are consists of "Cheremes", that is, hand posture (Dez), position (Tab) and motion (Sig). Thus, most of the notation system denotes signs as a series of combination of hand posture, orientation and position, although some of them denote 
motions as shape of trajectories. Sometimes several non-manual signals, such as facial expressions, are added on them.

HamNoSys [6], one of the most well-known sign notation system, also denote signs as a combination of hand posture, orientation and position. HamNoSys denote position of hands as combination of upper/middle/bottom of the shoulder and left and right of body, although it adds several more codes on update of version 4.0 , the resolution is still quite rough.

SignWriting [7], another one of the most well-known sign notation system, denotes hand posture and position, orientation and speed. As SignWriting is designed for easy denoting and reading of sign language and is derived from dance notation system, the notation is quite pictorial and subjective. Thus, automatic conversion from or to motion capture data seems quite difficult.

Kurokawa [8] developed a motion coding system for nonverbal interface and applied the coding system to denote sign language. However, resolution of the coding system, again, quite rough to synthesis signs animation from given code as the system designed mainly for motion recognition.

As discussed above, foregoing sign notation systems are based on subjective coding and its resolution are not detailed enough, new coding system, which mediates such subjective coding system and motion data, such as FACS [9] for facial expression, is essential for sign language information systems. As hand posture can be mapped via simple vector quantization as authors presented on previous conference and implemented on StrinGlove ${ }^{\circledR}[10]$, the coding system to denote hand position and orientation can be sufficient.

\section{Designing New Coordinate System}

\subsection{Basic Approach}

As discussed above, the target of this research is to develop hand posture/orientation coding system to mediate raw captured or kinematics data and existing subjective sign notation codes.

Careful observation of foregoing sign notation systems, especially new location symbols added on HamNoSys in version 4.0 [11], gives interesting insight of sign posture coordination. Figure 2 shows several new added location symbols. As shown in the figure, new version of HamNoSys gives locations of nominal hand by several body parts such as lip, ear lobe, nose and arms. On the other hand, quick review over several textbooks of sign languages also gives positions of hands or fingers by referring certain body parts. Therefore, human being seems to position hands relative to a certain body parts, such as lip, nose, chest, elbow, etc. Thus, coding system that let us denote relation between hands and other body parts can fit subjective insight of human beings as well as existing sign notation code.

On the other hand, to enable automatic conversion from or to "raw" motion data, such as captured motion data or generated kinematics data, the coding system must not be sparse or rough. The coding system should have enough resolution to show its precise position in a certain coordinate. 


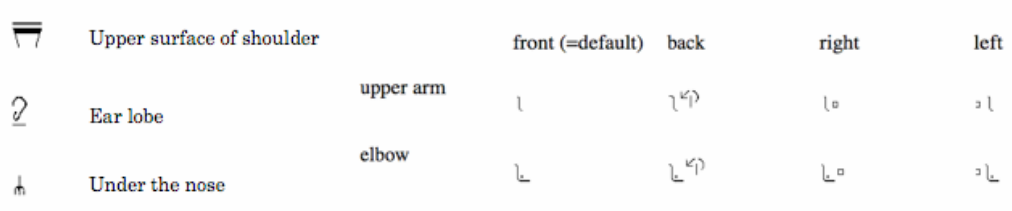

Fig. 2. New Location Symbols on HamNoSys 4.0 and its usage

The authors propose to develop new coordinate system suitable for sign motion rather than coding system. The coordinate system consists of sets of polar coordinate system whose origins are certain points (the reference points) on human body, such as "inner corner of right eyelid" or "eye". As the reference points are proportional to shape of body, the proposed system can proportion position and orientation of hands to various avatar models including cartoon-like model with big head as shown in Fig. 3.

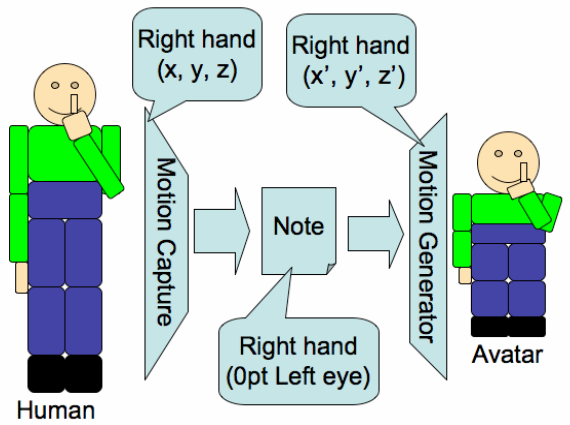

Fig. 3. Basic Concept of the Proposed Coordinate System

This coordinate system allows automatic conversion from "raw" motion data by calculating out distance of hand from several reference points and to "raw" motion data by mapping them on certain avatar. Additionally, a certain motion denoted on subjective notation code can be mapped onto the coordinate by giving distance on a certain rule.

\subsection{Picking Up the Reference Points}

To obtain reference points, this paper analyzes notations of Japanese - Japanese sign language dictionary issued by the Deaf association of Japan [12]. The dictionary has 8320 headwords (Japanese) and one headword has a pair of gestures (Japanese signs) and notation of each sign gestures in Japanese as shown in Fig. 4. In total, the dictionary has 16640 sign notations.

The authors scanned all pages of the dictionary and obtained notations via optical character reader. The obtained notations are analyzed through ChaSen, a morphological parser of the Japanese Language developed by NAIST Computer Linguistic Laboratory [13], which equips medical corpus developed for natural 


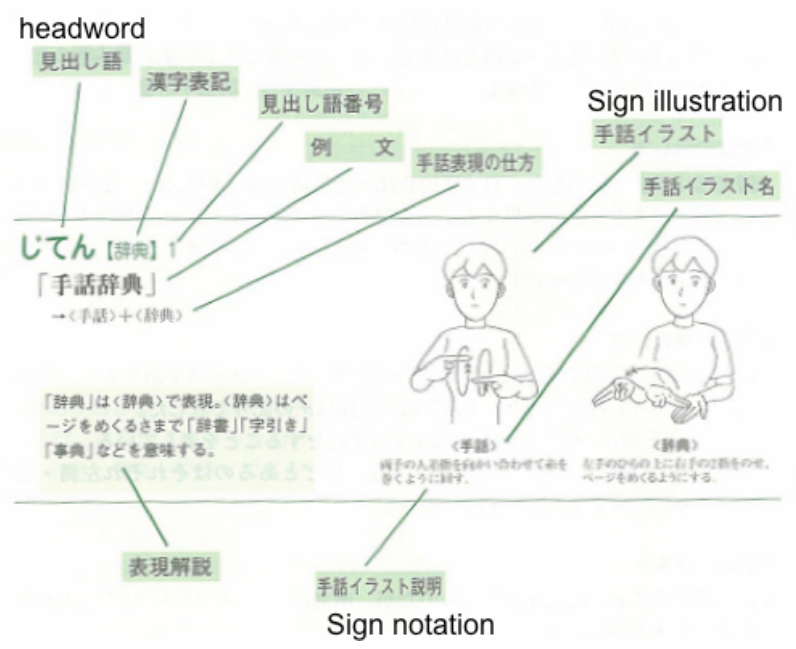

Fig. 4. Explanatory Notes of Japanese-Japanese Sign Dictionary [12]

language processing in medicine by the authors [14] to obtain proper anatomical nouns. Analysis extracted 43 candidates of reference points which can be successfully mapped on either a certain point of cinematic model or reference model of MPEG4 H-ANIM standard [15].

\subsection{Verification of Hypothesis}

The reference points are extracted based on a certain hypothesis, which a sign notation may gives positions of hands or fingers by referring certain body parts, obtained through quick review of sign textbooks. This section inspects the hypothesis through detailed analysis on sign documents.

As the sign notations obtained from the Japanese-Japanese sign language dictionary contains certain amount of error due to error of the optical character reader, this paper performs simple automatic correction before detailed analysis. As shown in Fig. 4, the dictionary uses one or two signs to denote one Japanese headword, and, as

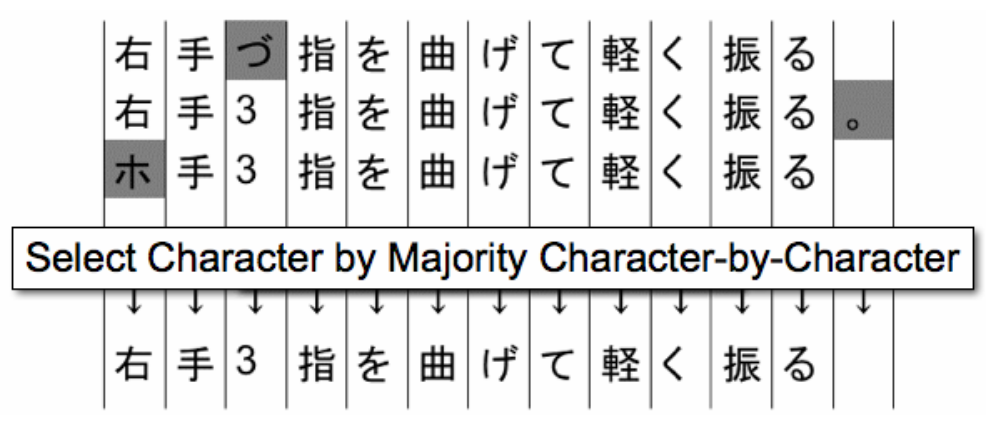

Fig. 5. Simple decision-by-majority algorithm 
a whole, 3235 signs are used to denote 8320 Japanese words. Therefore, most of the signs appear repeatedly. This paper compares multiple sign notations of a certain sign and decides character to pick by majority as shown in Fig. 5. This simple algorithm produces 2022 error-free sentences.

Selected 2022 sentences analyzed again via ChaSen with medical corpus. Through careful review on the result of morphological analysis, sentences with following features are marked as sentences supporting the hypothesis.

1. Sentence contains compound noun, which consists of body parts and postpositional modifier denoting position connected by possessive particle "NO", such as "yubi no saki (tip of finger)"'.

2. Sentences contains compound noun includes body parts followed by elative ("kara"), illative ("e") or inessive ("de") particles, such as "kuchimoto kara (from mouth)".

Tab. 1 shows the result. 301 sentences can be categorized as pattern 1 and 379 sentences can be categorized as pattern 2 . Thus, about $35 \%$ of the sentences denoted as the manner to support hypothesis.

Table 1. Analytical result

\begin{tabular}{|l|r|r|r|r|}
\hline & $\begin{array}{l}\text { Sentence with body } \\
\text { parts and } \\
\text { postpositional } \\
\text { modifier of position }\end{array}$ & $\begin{array}{l}\text { Sentence with body } \\
\text { parts followed with } \\
\text { elative/illative/inessive } \\
\text { particles }\end{array}$ & Others & Total \\
\hline Number of signs & 301 & 379 & 1342 & 2022 \\
\hline $\begin{array}{l}\text { Times to appear } \\
\text { in the dictionary }\end{array}$ & 1768 & 2507 & 7309 & 11665 \\
\hline
\end{tabular}

The result shows rest $65 \%$ of sentences doesn't have points to refer. However, it doesn't means these sentences doesn't fit with the hypothesis. In natural sign conversation, most of signs are shown in front of signer's chest, called neutral position, as it is most natural position to keep hands while moving. Quick review of rest $65 \%$ indicates most of the signs denoted without position are the signs shown at neutral position. So, more detailed analysis including idea of neutral position is required to verify the hypothesis.

\section{Discussion}

The analysis of Japanese-Japanese sign language gives 43 body positions, which can be mapped on H-ANIM standard avatar. Thus the coordinate system may denote given sign motion in a certain manner.

On the other hand, text analysis on source sign notation obtained from JapaneseJapanese sign language didn't clearly support the underlying hypothesis, as majority of source sign notations does not contains referring position. However, most of sign notations tacitly indicate that their target signs are shown around neutral position. For 
example, most of sign notations include information of motions, such as "move right hand upward" without denoting starting point, where the neutral position. Therefore, more detailed analysis is required to inspect whether the hypothesis is supported or not.

Although this paper proposed and mapped new coordinate system logically suitable for signs, sign language or human posture can be quite complicated. For example, to show "eye", a signer can indicate his/her eye from any direction of eye if the fingertip of index is $0 \mathrm{pt}$ (or small amount) from targeting eye. Thus, to define motion of sign posture, to define dominant part of hand is indispensable. However, as the dominant part can be defined as nearest point to the reference point, or dependent on hand posture itself, automatic conversion from "raw" data into the proposed coordinate may be realized to a certain extent.

\section{Conclusion}

This paper proposed new coordinate system suitable for sign animation, and defined possible origin of coordinates through morphological analysis of Japanese Sign Language Dictionary. The investigation clears that the proposed coordinate can proportional to avatar and be applicable for most of sign expressions. However, analysis on source sign notations cannot clearly support or decline the underlying hypothesis. More detailed analysis to verify the hypothesis is still open question. The authors are developing coordinate conversion to evaluate availability of the proposed coordinate system.

Acknowledgements. This research is partly supported by Grant-in-Aid for Young Scientists (A) (17680008) from The Ministry of Education, Culture, Sports, Science and Technology, Japan.

\section{References}

1. Nagashima, Y., Kanda, K.: Present State and Issue of Sign Linguistic Engineering. In: Proc. HCI Int. vol. 3, pp. 387-391 (2001)

2. Kuroda, T., Sato, K., Chihara, K.: S-TEL an Avatar Based Sign Language Telecommunication System. Int, J. Virtual Reality 3(4), 21-27 (1998)

3. Information Technology - Coding of Audio-Visual Objects- Part2: Visual. ISO/IEC 14496-2 (2001)

4. Kuroda, T., Makari, W., Manabe, Y., Chihara, K.: Transformation of Signing Avatar to Fit User's Figure. Moroccan J. Cont. Comp. Sci. and Sig. Proc. 3(1) (2001)

5. Stokoe, W.C., Casterline, D., Croneberg, C.: A Dictionary of American Sign Language on Linguistic Principles. Gallaudet University Press, Washington DC (1965)

6. Hanke, T.: HamNoSys; Representing Sign Language Data In Language Resources and Language Processing Contexts. In: Proc. LREC, pp. 1-6 (2004)

7. Sutton, V.: The SignWriting Literacy Project. In: Proc. Cognition AREA Conf. (1998)

8. Kurokawa, T.: Gesture Coding and a Gesture Dictionary for a Nonverbal Interface. IEICE Trans. Fund. Elec. 75, 112-121 (1992) 
9. Ekman, P., Friesen, W.V.: Facial Action Coding System: a Technique for the Measurement of Facial Movement. Consulting Psychologists Press, Palo Alto (1978)

10. Kuroda, T., Tabata, Y., Goto, A., Ikuta, H., Murakami, M.: Consumer Price Data-Glove for Sign Language Recognition. In: Proc. ICDVRAT, pp. 253-258 (2004)

11. Schmaling, C., Hanke, T.: HamNoSys 4.0. (2004) http://www.sign-lang.uni-hamburg.de/Projekte/HamNoSys/HNS4.0/englisch/HNS4.pdf

12. Yonekawa, A., (eds.): Japanese-Japanese Sign Language Dictionary. Japanese Federation of the Deaf, Tokyo (1997)

13. Matsumoto, Y., Kitauchi, A., Yamashita, T., Hirano, Y., Matsuda, H., Takaoka, K., Asahara, M.: Moriphological Analysis System ChaSen Manual. Nara Institute of Science and Technology (2001)

14. Takemura, T., Ashida, S.: A Concept of Medical Corpus for Natural Language Processing in Medicine. In: Proc. Int. Work. Enterprise Networking Comp. in Health Care Industry (2001)

15. Kuroda, T., Kazuya, O., Takemura, T., Nagase, K., Yoshihara, H.: New Coordinate System Suitable for Sign Animation. Int. J. Disability and Human Development 5(2), 113 117 (2006) 